des als Ressource im Parteienwettbewerb sind seine traditionellen Risiken: Korruptionsgefahr, verzerrte Wettbewerbschancen der Parteien und Umsetzung sozialer Ungleichheit in politische Entscheidungen. Beim Kampf gegen diese Risiken demokratischer Politik spielt Transparenz der Politikfinanzierung eine zentrale Rolle.

Gerade ein an Österreich wenig interessierter Leser findet in den abschließenden (durchaus landesbezogen formulierten) Kapiteln eine Fülle von Ansatzmöglichkeiten für die Betrachtung des eigenen Landes oder vergleichende Analysen. Zunächst werden Maßstäbe vorgeführt, deren Einsatz helfen kann, die tatsächlichen Kosten der Parteiendemokratie differenziert zu beurteilen. Dekonzentration des Parteiensystems, Bedeutungsverlust der Parteiorganisationen, Bedeutungszuwachs der Medien und veränderte Medienlandschaft sind keine Besonderheiten der österreichischen Politik, wirken aber auf die Politikfinanzierung ein. Daraus werden oft Sachzwänge für die Kostenentwicklung abgeleitet. Sickinger vermeidet solche Pauschalthesen und zeigt ein Primat der Politik (und damit Handlungsmöglichkeiten) für die tatsächliche Ausgestaltung der Politikfinanzierung auf. Ausführlich und differenziert setzt er sich mit den vielfach behaupteten Auswirkungen der öffentlichen Parteienfinanzierung auseinander. Als Verfestigung des Parteiensystems konstatiert er vorrangig ein Festhalten am parteiorganisatorischen Status quo. Die finanzielle Autonomie der Parteileitungen (von den Parteimitgliedern) ist aber auch eine verstärkte Orientierung der Parteieliten an der Wählerschaft. Wichtige Änderungen des Parteialltags (nämlich Professionalisierung und Bürokratisierung) werden allerdings vom Konstrukt Etatisierung nicht gemessen. Gegen die Korruptionsgefahr ist öffentliche Parteienfinanzierung nur hilfreich im Zusammenwirken mit öffentlicher Kontrolle und einer medienvermittelten Abschreckung durch politische Skandale. Kurzum: Als Problemlösung ist die staatliche Politikfinanzierung nur ein möglicher Faktor, der vor allem verstärkend wirken kann.

Karl-Heinz Naßmacher

\title{
Gewichtiger Jubiläumsband zu Extremismus und Demokratie
}

Backes, Uwe und Eckhard Jesse (Hrsg.): Jahrbuch Extremismus \& Demokratie 2008, 20. Jahrgang, Nomos Verlagsgesellschaft, Baden-Baden 2009, 486 Seiten, € 49,-.

Der 20. Band des Jahrbuches „Extremismus und Demokratie“ stellt einen wahren Jubiläumsband dar: Das „60/20“-Jubiläum von provisorischer Reorganisation des Staates im Westen und friedlicher Revolution im Osten setzt den Rahmen. Außerdem wird Bezug genommen auf die Studentenunruhen vor 40 Jahren, auf die 30. Wiederkehr des „deutschen Herbstes“ und die 25 Jahre zurückliegende Gründung der „Marxistisch-Leninistischen Partei Deutschlands“. Die 486 Seiten teilen sich in 97 Seiten reflektierende „Analysen“, 137 Seiten faktenreiche „Daten, Dokumente, Dossiers“ und einen umfangreichen Literaturteil (212 Seiten), ergänzt um Einführung und Personenregister. Wiederum sind sowohl Nachwuchskräfte als auch namhafte Wissenschaftler als Autoren gewonnen worden. Erfreulicherweise verwendet das Jahrbuch weiterhin die herkömmliche Orthographie.

Zwei überblicksartig angelegte Artikel der Herausgeber dokumentieren 60 Jahre Bundesrepublik Deutschland als Periode extremistischer Herausforderungen einerseits, 
funktionierender Parteiendemokratie andererseits: Uwe Backes differenziert die Entwicklung systemoppositioneller Gruppen nach Raum, Zeit und ideologischer Ausrichtung, wobei die Gewichtung einen deutlichen Aktualitätsbezug setzt. Lässt man den islamistischen Fundamentalismus beiseite, erweist sich der innerdeutsche Ost-West-Vergleich einmal mehr als fruchtbar, da sowohl die als „semiloyal“ charakterisierte PDS/Linke als auch die „aggressiv-verfassungsfeindliche“ NPD in den neuen Bundesländern bislang ein für sie günstigeres Terrain vorfinden als im Westen. Nur bleibt bei Backes institutionenbezogen die Differenzierung zwischen „tolerierten“ und „gestützten“ Minderheitsregierungen - relevant für Sachsen-Anhalt - leider unberücksichtigt.

Eckhard Jesses Darstellung der Demokratieentwicklung unterscheidet zeitlich die Phasen vor und nach der Wiedervereinigung, orientiert sich ansonsten aber stärker an theoretischen Erklärungsmodellen für das Parteiensystem. Im Unterschied zu Peter Haungs zeigt Jesse dabei keine grundsätzliche Abneigung gegen den Parteienstaatsbegriff, wenn nur dessen Abgrenzung von der Leibholz'schen Variante, heute von Karl-Rudolf Korte vertreten, eindeutig ist ${ }^{1}$. Für den Grenzbereich zwischen Demokratie und Extremismus weist Jesse zutreffend auf das Opportunitätsprinzip bei der Beantragung eines Parteiverbotes hin. Diese Praxis außerrechtlicher Toleranz hat zur Folge, dass nicht jede „nicht-verbotene“ Partei "per se demokratisch“ ist, was Jesse zu Recht auch öffentlich herausgestellt sehen möchte (S. 49). Nicht erkennbar wird, dass es in Bezug auf die Gruppierungen Freiheitliche Deutsche Arbeiterpartei (FAP) und Nationale Liste (NL) nicht bei der Erwägung eines Parteiverbots (S. 48) geblieben, sondern zu einer Antragstellung gekommen ist - trotz Unzuständigkeit des Bundesverfassungsgerichts ${ }^{2}$ letztlich sogar mit dem gewünschten Erfolg.

Deutlich herausgearbeitet werden die unterschiedlichen Bedingungen für die Entstehung von Parteienkonkurrenzverhältnissen in West und Ost und die von Jesse für wahrscheinlich gehaltene Zunahme zentrifugaler Kräfte im Parteiensystem - wobei die Prognose unter Einbeziehung der Wandlung des Parteiensystems zwischen 1949 und 1953 (hierzu Wilhelm Hennis) positiver, unter Berücksichtigung der Analysen Giovanni Sartoris zur Weimarer Republik negativer ausfallen könnte ${ }^{3}$.

Der Artikel Werner Müllers über Karl Schirdewan führt zurück in die DDR der 50er Jahre. Anhand der in jüngerer Zeit zugänglich gewordenen Quellen gelingt es Müller, die Selbststilisierung Schirdewans zum Oppositionellen gegen die SED-Diktatur unter Walter Ulbricht zu erschüttern und ihn als stalinistischen Funktionär zu demaskieren. Schirdewans spätere Berufung in den „Rat der Alten“ der PDS wirft daher Fragen zur Erneuerung dieser Partei auf.

Wolfgang Kraushaar kommt mit seinem informativen Artikel zu den „Achtundsechzigern“ Extremismus und Demokratie in demselben Text nahe. Eigenartigerweise finden sich dort sachlich unzutreffende Begriffe wie „Radikalenerlaß“ und „soziale Widersprüche“. Dabei gab es keine eigenmächtig-autoritative Entscheidung administrativer Stellen, durch die

1 Vgl. Gerhard Leibholz, Strukturprobleme der modernen Demokratie, Frankfurt am Main 1974, S. 78 - 129; Peter Haungs, Parteiendemokratie in der Bundesrepublik Deutschland, Berlin 1980, S. 12 - 25; Karl-Rudolf Korte / Manuel Fröhlich, Politik und Regieren in Deutschland, Paderborn 2004, S. 93.

2 Vgl. BVerfGE 91, S. 262 (S. 276 ff.).

3 Vgl. Wilhelm Hennis, Parteienstruktur und Regierbarkeit, in: ders. I Peter Graf Kielmansegg / Ulrich Matz (Hrsg.), Regierbarkeit, Bd. 1, Stuttgart 1977, S. 150 - 195, S. 178 f.; Giovanni Sartori, Parties and Party Systems, Cambridge 1976, S. 155 f. 
Grundrechte entzogen oder eingeschränkt worden wären, und der Ausdruck „Widerspruch“ gehört in den Bereich der Logik, wo er eine Unvereinbarkeit bezeichnet, die notwendigerweise auszuräumen ist, nicht in die soziale Realität, wo „Gegensätze“ existieren, die in praxi miteinander ausgeglichen werden können.

Beiträge im Dokumentationsteil ergänzen Backes' und Jesses langfristige Analysen um aktuelle Details: Jesse bietet eine Auswertung der Bremer Bürgerschaftswahl von 2007, Backes liefert organisationsbezogene Einblicke in Randbereiche des politischen Spektrums und deren Gewaltbereitschaft, wie sie sich 2007 beim G8-Gipfel von Heiligendamm gezeigt hat.

Sandra Pingel-Schliemanns Untersuchung zur NPD im Landtag von Mecklenburg-Vorpommern lässt einen „antifaschistischen“ Konsens der übrigen Fraktionen erkennen. Die Abhandlung bezieht neben Parlaments- auch Parteiaktivitäten im Lande ein. Helmut Müller-Enbergs, nach eigenem Bekenntnis in seiner Jugend als Mitglied der Nachwuchsorganisation Revolutionärer Jugendverband Deutschlands (RJVD) 1977 bis 1979 in Recklinghausen selbst ein „Insider“ der Szene gewesen (S. 180), bietet detaillierte Informationen zur Politsekte Marxistisch-Leninistische Partei Deutschlands (MLPD), die wechselnde Organisationsstrukturen und konspirative Elemente, Konstanz der Führung und politische Einflusslosigkeit - trotz finanzieller Potenz - miteinander verbindet. Streckenweise ähnlich wie Christian Menhorn 2008 stellt Marc Brandstetter auffällige Parallelen zwischen militanten rechts- und linksextremen Gruppierungen dar, die durch Übernahme linker Praktiken seitens rechter Subkulturen entstanden sind.

Die personale Dimension der Politik, verdienstvollerweise erneut berücksichtigt, ist durch ein breit angelegtes Portrait des Rechtsextremisten Christian Worch präsent, zu dem der Autor, Martin Thein, sprachlich wie inhaltlich den nötigen Abstand hält. Eine Länderstudie, diesmal von Melani Barlai und Florian Hartleb zu Ungarn, zeigt ein Land mit einem stabilen Parteiensystem, jedoch erheblichem rechten Potential, das sich aus mehreren Quellen speist und gesellschaftlich als „Bewegung“ in Erscheinung tritt.

Norman Bock liefert als Zeitschriftenportrait eine Analyse zur „Jungen Welt“ - einem Blatt, das eine Vielzahl von Redakteuren insbesondere auch in leitenden Stellungen aufweist, die dem Unterdrückungs- und Spionageapparat des SED-Regimes gedient haben.

Im umfangreichen Literaturteil des Jahrbuches stellt Eckhard Jesse in seinem kenntnisreichen Besprechungsaufsatz zum „deutschen Herbst“ von 1977 fest: „Das Thema Terrorismus floriert“. Alexander Gallus nimmt sich der Intellektuellen an, deren Gefährdung durch totalitäre Ideologien herausgearbeitet wird. Fünf Autoren diskutieren Richard Dawkins " Der Gotteswahn“, wobei die Ansichten zwischen „populistisch-verfehlt" und der Konzedierung eines trotz ungeeigneter Form diskussionswürdigen Inhalts liegen. Fragen nach der von Dawkins anvisierten Welt - ohne zahlreiche Kunstwerke, Aufopferungsbereitschaft in Dienst und Widerstand und ohne Verantwortung gemäß christlicher Vorstellung (2. Kor. 5, 10; Mt. 25, 31 - 46) - werden dabei noch nicht einmal in den Blick genommen. Christopher Daase schließlich setzt sich kritisch mit der von Michael Mann aufgestellten These auseinander, ethnische Säuberungen seien als „dunkle Seite der Demokratie“ aufzufassen.

Zahlreiche weitere Rezensionen und Kurzinformationen vermitteln dem Leser Orientierung über vielfältige Neuerscheinungen. Wie seine Vorgänger bietet damit auch der 20 . Band wertvolle Kenntnisse und Interpretationen zu einem breiten, jedoch häufig vernachlässigten Themengebiet. 